\title{
Avaliação de medidas da persistência da lactação de cabras da raça Saanen sob modelo de regressão aleatória
}

\author{
Gilberto Romeiro de Oliveira Menezes ${ }^{1}$, Robledo de Almeida Torres ${ }^{2}$, José Lindenberg \\ Rocha Sarmento ${ }^{3}$, Marcelo Teixeira Rodrigues ${ }^{2}$, Ana Lúcia Puerro de Melo ${ }^{4}$, Felipe Gomes \\ da Silva ${ }^{5}$, Luiz Fernando Brito ${ }^{6}$
}

\footnotetext{
${ }^{1}$ Pós-Graduação em Genética e Melhoramento, UFV. Bolsista do CNPq.

2 Departamento de Zootecnia, UFV. Bolsista do CNPq.

${ }^{3}$ Departamento de Zootecnia, UFPI.

${ }^{4}$ Pós-Graduação em Zootecnia, UFV. Bolsista da CAPES.

${ }^{5}$ Graduação em Agronomia, UFV.

${ }^{6}$ Graduação em Zootecnia, UFV. Bolsista de Iniciação Científica do CNPq.
}

RESUMO - Utilizaram-se 10.238 registros semanais de produção de leite no dia do controle, provenientes de 388 primeiras lactações de cabras da raça Saanen, na avaliação de seis medidas da persistência da lactação, a fim de verificar qual a mais adequada para o uso em avaliações genéticas para a característica. As seis medidas avaliadas são adaptações de medidas utilizadas em bovinos de leite, obtidas por substituir, nas fórmulas, os valores de referência de bovinos pelos de caprinos. Os valores usados nos cálculos foram obtidos de modelos de regressão aleatória. As estimativas de herdabilidade para as medidas de persistência variaram entre 0,03 e 0,09 . As correlações genéticas entre medidas de persistência e produção de leite até 268 dias variaram entre -0,64 e 0,67. Por apresentar a menor correlação genética com produção aos 268 dias $(0,14)$, a medida de persistência $\mathrm{PS}_{4}$, obtida pelo somatório dos valores do $41^{0}$ ao $240^{0}$ dia de lactação como desvios da produção aos 40 dias de lactação, é a mais indicada em avaliações genéticas para persistência da lactação em cabras da raça Saanen. Assim, a seleção de cabras de melhor persistência da lactação não altera a produção aos 268 dias. Em razão da baixa herdabilidade dessa medida $(0,03)$, pequenas respostas à seleção são esperadas neste rebanho.

Palavras-chave: correlação genética, leite, pico de lactação, seleção

\section{Evaluation of persistency lactation measures of Saanen goats under random regression model}

\begin{abstract}
It was used 10,238 weekly milk production records on the control day from the first 388 lactations of Saanen goats on the evalution of six lactation persistency measures in order to find out which was the best fitted for using in genetic evaluations on this trait. These six evaluated measures are adaptations from those used on dairy cattle, obtained by replacing, in the formula, bovine reference values by the goat ones. The values used in the calculations were obtained from random regression models. Heritability estimates for persistency measures ranged from 0.03 to 0.09 . Genetic correlations between persistency measures and milk production until 268 days ranged from -0.64 to 0.67 . Because it presented the lowest genetic correlation with production at 268 days $(0.14)$, the persistency measure $\mathrm{PS}_{4}$ obtained by the sum of the genetic values from the $41^{\text {st }}$ to the $240^{\text {th }}$ day of lactation as deviations of production at 40 days of lactation, is the best recommended for genetic evaluations of lactation persistence on Saanen goats. Thus, the selection of the goats with the best persistency of lactation does not change the production at 268 days. Due to the low heritability of this measure (0.03), few responses to selection are expected on this herd.
\end{abstract}

Key Words: genetic correlation, lactation peak, milk, selection

\section{Introdução}

O sucesso de sistemas de produção animal é mundialmente dependente do grau de eficiência econômicoprodutiva que se atinge, sendo importante salientar que essa eficiência não significa, propriamente, máxima produtividade. A cadeia de produção de leite caprino não é diferente e, portanto, precisa estar alinhada a essa tendência mundial. A busca, por meio do melhoramento genético, de animais mais eficientes e não apenas os mais produtivos certamente é uma estratégia a ser seguida. Esforços nesse sentido, em bovinos de leite, têm sido feitos pela seleção 
para características funcionais, as quais estão intimamente ligadas à eficiência de produção (Cobuci et al., 2007). Dentre tais características, a persistência da lactação tem recebido atenção especial dos pesquisadores. Todavia, em caprinos, há escassez de estudos relacionados ao tema.

Segundo Cobuci et al. (2003), a persistência da lactação é definida como a capacidade do animal em manter sua produção de leite após atingir sua produção máxima na lactação, sendo que maiores persistências da lactação são consideradas vantajosas, pois levam à redução nos custos com alimentação (Sölkner \& Fuchs, 1987), diminuição de incidência de doenças metabólicas e de membros locomotores (Harder et al., 2006) e melhoria de fertilidade (Lean et al., 1989).

Na literatura são encontrados basicamente quatro tipos de mensuração da persistência da lactação: mensurações baseadas em razões entre produção de leite, em diferentes fases da lactação; na variação da produção de leite; em parâmetros de modelos não lineares; e nos valores genéticos obtidos por meio de coeficientes dos modelos de regressão aleatória, sendo que estes últimos têm sido indicados como os mais adequados em vários trabalhos com bovinos de leite (Jamrozik et al., 1997; Cobuci et al., 2004; Cobuci et al., 2006b; Cobuci et al., 2007).

Diferentes medidas da persistência baseadas nos valores genéticos por modelos de regressão aleatória foram propostas para bovinos de leite (Jamrozik et al., 1997; Jakobsen et al., 2002; Cobuci et al., 2004; Cobuci et al., 2006b; Herrera et al., 2008). No entanto, para caprinos nada foi encontrado, o que indica a necessidade de estudos que venham a propor formas de avaliar a persistência da lactação de cabras leiteiras por modelos de regressão aleatória.
Neste contexto, foram avaliadas diversas medidas da persistência da lactação de cabras da raça Saanen por modelos de regressão aleatória, a fim de se encontrar aquela mais adequada para o uso em avaliações genéticas para essa característica.

\section{Material e Métodos}

Analisaram-se 10.238 registros semanais de produção de leite no dia do controle, provenientes de 388 primeiras lactações de cabras da raça Saanen, divididas em dois grupos genéticos: puro Saanen (243 cabras - 62,6\%) e 1/4 a $1 / 2$ Saanen ( 145 cabras - 37,4\%).

Os controles foram registrados semanalmente, oriundos de ordenha mecânica, realizada duas vezes ao dia. Os animais foram mantidos em baias coletivas sob o sistema de estabulação livre. Os dados foram coletados entre os anos de 1999 e 2007. O banco de dados inicial era composto por 17.104 registros de produção de leite no dia do controle, porém apenas foram considerados os registros de controles da $2^{\text {a }}$ até a 39ạ semana de lactação. Além disso, foram excluídos da análise animais com produções superiores ou inferiores a três desvios-padrão da média da semana da lactação, com idade ao parto inferior a 10 meses ou superior a 30 meses, produções que ocorreram em grupos contemporâneos (anoquinzena do controle) com menos de três cabras e lactações com menos de seis controles. Os grupos contemporâneos foram formados pela alocação dos registros de produção de leite no dia do controle dentro de classes formadas pelo ano e pela quinzena dentro do respectivo ano do controle (Tabela 1).

A matriz dos numeradores dos coeficientes de parentesco continha 538 animais, sendo 150 sem registro de antecedentes.

Tabela 1 - Produção de leite na semana do controle da primeira lactação de cabras da raça Saanen

\begin{tabular}{|c|c|c|c|c|c|c|c|}
\hline Semana & № de registros & Média (kg) & Desvio-padrão & Semana & № de registros & Média (kg) & Desvio-padrão \\
\hline 2 & 197 & 1,88 & 0,65 & 21 & 303 & 1,80 & 0,80 \\
\hline 3 & 248 & 2,06 & 0,77 & 22 & 280 & 1,69 & 0,76 \\
\hline 4 & 283 & 2,15 & 0,80 & 23 & 269 & 1,73 & 0,72 \\
\hline 5 & 280 & 2,21 & 0,83 & 24 & 256 & 1,75 & 0,77 \\
\hline 6 & 335 & 2,22 & 0,82 & 25 & 302 & 1,61 & 0,71 \\
\hline 7 & 324 & 2,20 & 0,81 & 26 & 288 & 1,50 & 0,64 \\
\hline 8 & 280 & 2,20 & 0,83 & 27 & 292 & 1,58 & 0,72 \\
\hline 9 & 330 & 2,21 & 0,88 & 28 & 277 & 1,52 & 0,67 \\
\hline 10 & 343 & 2,17 & 0,86 & 29 & 253 & 1,44 & 0,69 \\
\hline 11 & 340 & 2,17 & 0,95 & 30 & 229 & 1,51 & 0,68 \\
\hline 12 & 325 & 2,08 & 0,85 & 31 & 240 & 1,47 & 0,66 \\
\hline 13 & 286 & 2,21 & 0,88 & 32 & 254 & 1,43 & 0,71 \\
\hline 14 & 308 & 2,12 & 0,87 & 33 & 243 & 1,41 & 0,72 \\
\hline 15 & 332 & 2,05 & 0,84 & 34 & 202 & 1,39 & 0,66 \\
\hline 16 & 286 & 2,12 & 0,81 & 35 & 190 & 1,48 & 0,72 \\
\hline 17 & 315 & 1,91 & 0,81 & 36 & 172 & 1,56 & 0,72 \\
\hline 18 & 301 & 1,88 & 0,83 & 37 & 170 & 1,40 & 0,70 \\
\hline 19 & 293 & 1,91 & 0,84 & 38 & 168 & 1,41 & 0,73 \\
\hline 20 & 298 & 1,91 & 0,75 & 39 & 146 & 1,49 & 0,71 \\
\hline
\end{tabular}


A modelagem da curva média de lactação da população e das curvas aleatórias genética aditiva e de ambiente permanente foi feita usando a função de Wilmink modificada ( $\left.y=a_{1}+a_{2} t+a_{3} e^{-0,035 t}\right)$, cuja mudança consistiu em usar o valor $-0,035$ em vez de -0,05 no termo exponencial da função assumindo-se heterogeneidade de variância residual com seis classes de resíduo ao longo da curva de lactação $\left(\sigma_{e 1}^{2}, \sigma_{e 2}^{2}, \sigma_{e 3}^{2}\right.$, $\sigma_{e 4}^{2}, \sigma_{e 5}^{2}$ e $\sigma_{e 6}^{2}$ ), agrupadas da seguinte forma: $2^{\underline{a}}$ a $7 \underline{a}$, $8^{\underline{a}}$ a $13^{\underline{a}}, 14^{\underline{a}}$ a $19^{\underline{a}}, 20^{\underline{a}}$ a $26^{\underline{a}}, 27^{\underline{a}}$ a $32^{\underline{a}}$ e $33^{\underline{a}}$ a $39^{\underline{a}}$ semana de lactação (Menezes, 2008).

O modelo de regressão aleatória aplicado aos registros de produção de leite no dia do controle foi:

$\mathrm{y}_{\mathrm{ij}}=\mathrm{F}+\sum_{m=1}^{3} b_{m} Z_{m}\left(t_{i j}\right)+\sum_{m=1}^{3} a_{i m} Z_{m}\left(t_{i j}\right)+\sum_{m=1}^{3} p_{i m} Z_{m}\left(t_{i j}\right)+e_{i j}$ em que: $\mathrm{y}_{\mathrm{ij}}=$ produção de leite no controle $\mathrm{j}$ da cabra $\mathrm{i}$; $\mathrm{F}$ = conjunto de efeitos fixos, constituído por grupo contemporâneo (ano-quinzena do controle - 176 subclasses), grupo genético (puro e $1 / 4$ a $1 / 2$ Saanen), tipo de parto (simples e duplo) e pela covariável idade da cabra no dia do controle e efeitos linear e quadrático; $\mathrm{b}_{\mathrm{m}}=\mathrm{m}^{\text {ésimo }}$ coeficiente de regressão fixa da produção no dia do controle sobre a semana de lactação; $\mathrm{a}_{\mathrm{im}}$ e $\mathrm{p}_{\mathrm{im}}$ = coeficientes de regressão genético aditivo e de ambiente permanente, respectivamente, associados aos coeficientes específicos da função de Wilmink modificada, que podem ser representados por $\mathrm{Z}_{\mathrm{m}}^{\prime}=\left(\mathrm{Z}_{1} \mathrm{Z}_{2} \mathrm{Z}_{3}\right)^{\prime}=(1 \mathrm{t} \mathrm{d})^{\prime}$, de modo que $\mathrm{d}=\mathrm{e}^{-0,035 \mathrm{t}}$; t e $\mathrm{m}$ = respectivamente, o período de dias em lactação após o parto e o mésimo parâmetro da função; $\mathrm{e}_{\mathrm{ij}}=$ erro aleatório associado a cada controle j da cabra i. A função de Wilmink também foi usada para o ajuste da curva fixa (média populacional).

As soluções para os coeficientes de regressão aleatória genéticos aditivos de cada animal i são dadas pelo vetor $\hat{a}^{\prime}{ }_{i}=\left(\hat{a}_{1 \mathrm{i}} \hat{a}_{2 \mathrm{i}} \hat{a}_{3 \mathrm{i}}\right)$, entretanto, isoladamente, não são suficientes para classificar ou selecionar os animais. No entanto, funções dessas soluções fornecem importantes informações para os procedimentos de seleção. Assim, o valor genético aditivo esperado do animal i, no período de lactação t, foi obtido por:

$$
\mathrm{V}_{\mathrm{gt}}=\left(\hat{\mathrm{a}}_{1 \mathrm{i}}+\hat{\mathrm{a}}_{2 \mathrm{i}} \mathrm{t}+\hat{\mathrm{a}}_{3 \mathrm{i}} \mathrm{d}\right)=\mathrm{Z}_{\mathrm{m}}^{\prime} \hat{\mathrm{a}}_{\mathrm{i}},
$$

em que $Z_{m}^{\prime}=(1 \mathrm{td})$ '.

O valor genético para produção de leite, considerando a lactação completa (em 268 dias, correspondendo, aproximadamente a 9 meses), $\mathrm{Vg}_{268}$, a partir da produção de leite no dia do controle, foi obtido pela soma dos valores genéticos de cada período (t) de dias em lactação do animal i:

$$
V \mathrm{~g}_{268}=\sum_{t=10}^{268} V g_{t}=\left[\begin{array}{lll}
259 & 36001 & 20,4860
\end{array}\right] * \hat{\mathrm{a}}_{\mathrm{i}}
$$

Nas análises, foram considerados os controles entre o $10^{0}$ e $268^{\circ}$ dia de lactação após a data do parto, devido à ausência dos controles entre o $1^{\underline{0}}$ e $9 \underline{0}$ dia de lactação.

As medidas de persistência da lactação de cabras avaliadas sob modelo de regressão aleatória foram adaptações de medidas encontradas na literatura de bovinos de leite, não tendo sido encontrados estudos sobre o tema com caprinos. As adaptações consistiram em substituir, nas fórmulas (medidas), os valores referência de bovinos pelos de caprinos. Assim, considerouse uma duração de lactação de 268 dias (9 meses), em vez de 305 dias (bovinos), e um pico de lactação aos 40 dias (Tabela 1), em vez dos 60 dias adotados para bovinos. Outra adaptação foi considerar o primeiro controle leiteiro aos 10 dias, enão aos seis dias pós-parto.

A primeira medida da persistência na lactação, $\mathrm{PS}_{1}$, é dada pela diferença entre valores genéticos preditos para produção de leite aos $240^{\circ}$ e $40^{\circ}$ dia de lactação:

$$
\mathrm{PS}_{1}=\left(V g_{240}-V g_{40}\right)=\left[\begin{array}{lll}
0 & 200 & -0,24637
\end{array}\right] * \hat{\mathrm{a}}_{\mathrm{i}}
$$

A segunda, $\mathrm{PS}_{2}$, foi obtida pela diferença entre as áreas sob a curva de lactação, nos terços mediano e inicial da lactação (adaptada de Jakobsen et al., 2002):

$$
\mathrm{PS}_{2}=\left(\sum_{t=96}^{181} V g_{t}-\sum_{t=10}^{95} V g_{t}\right)=\left[\begin{array}{ll}
0 & 7396-18,51831
\end{array}\right] * \hat{\mathrm{a}}_{\mathrm{i}}
$$

A terceira, $\mathrm{PS}_{3}$, foi obtida pela área sob a curva de lactação, no terço final da lactação, subtraída pela área sob a curva, no terço inicial da lactação (adaptada de Jakobsen et al., 2002):

$$
\mathrm{PS}_{3}=\left(\sum_{t=182}^{268} V g_{t}-\sum_{t=10}^{95} V g_{t}\right)=\left[\begin{array}{ll}
1 & 15060-19,43102
\end{array}\right] * \hat{\mathrm{a}}_{\mathrm{i}}
$$

A quarta, $\mathrm{PS}_{4}$, foi obtida pelo somatório dos valores genéticos, no período do $41^{\circ}$ ao $240^{\circ}$ dia de lactação, como desvios da produção aos 40 dias de lactação (adaptada de Jamrozik et al., 1997):

$$
\mathrm{PS}_{4}=\sum_{t=41}^{240}\left(V g_{t}-V g_{40}\right)=\left[\begin{array}{ll}
0 & 20100-42,40270
\end{array}\right] * \hat{\mathrm{a}}_{\mathrm{i}}
$$

A quinta, $\mathrm{PS}_{5}$, foi obtida pelo somatório das contribuições de cada dia de produção $\left(\mathrm{Vg}_{\mathrm{t}}\right)$ no período do $40^{\circ}$ ao $239^{\circ}$ dia de lactação, como desvios da produção aos 240 dias de lactação (adaptada de Jakobsen et al., 2002):

$$
\mathrm{PS}_{5}=\sum_{t=40}^{239}\left(V g_{t}-V g_{240}\right)=\left[\begin{array}{lll}
0 & 201007,11813
\end{array}\right] * \hat{\mathrm{a}}_{\mathrm{i}}
$$

A sexta, $\mathrm{PS}_{6}$, é uma modificação da primeira medida da persistência da lactação $\left(\mathrm{PS}_{1}\right)$, tendo sido obtida pela diferença entre valores genéticos preditos para produção de leite aos 250ㅇ e 70으 dia de lactação (adaptada de Cobuci et al., 2004):

$$
\mathrm{PS}_{6}=\left(V g_{250}-V g_{70}\right)=\left[\begin{array}{lll}
0 & 180-0,08613
\end{array}\right] * \hat{\mathrm{a}}_{\mathrm{i}}
$$

Todos os resultados basearam-se nas expressões genéticas de $\mathrm{PS}_{\mathrm{i}}$, as quais são obtidas pela aplicação do MRA nas produções de leite no dia do controle. 
Menores valores de $\mathrm{PS}_{1}, \mathrm{PS}_{2}, \mathrm{PS}_{3}, \mathrm{PS}_{4}$ e $\mathrm{PS}_{6}$ indicam maiores níveis de persistência da lactação, enquanto maiores valores de PS $_{5}$ indicam maiores níveis de persistência da lactação (Jakobsen et al., 2002; Cobuci et al., 2004).

As covariâncias entre os coeficientes de regressão aleatória genéticos aditivos e de ambiente permanente foram estimadas pelo método da máxima verossimilhança restrita livre de derivadas (REML), usando o programa DXMRR do DFREML (Meyer, 1998). A maximização da função de verossimilhança foi realizada usando o algoritmo Powell (Powell, 1964). O critério de convergência utilizado foi $10^{-9}$.

As estimativas de variâncias genéticas $\left(\hat{\sigma}_{a}^{2}\right)$ e de ambiente permanente ( $\hat{\sigma}_{p}^{2}$ ) para produção de leite em um período t qualquer da lactação são dadas por:

$$
\begin{gathered}
\hat{\sigma}_{a}^{2}=\mathrm{Z}_{\mathrm{m}}{ }^{*} \hat{K}_{a} * \mathrm{Z}_{\mathrm{m}} \\
\hat{\sigma}_{p}^{2}=\mathrm{Z}_{\mathrm{m}}^{\prime} * \hat{K}_{p} * \mathrm{Z}_{\mathrm{m}}
\end{gathered}
$$

em que $\hat{K}_{a}$ e $\hat{K}_{p}$ são matrizes das estimativas de variâncias e covariâncias entre os coeficientes de regressão aleatória genéticos e de ambiente permanente, respectivamente; $\mathrm{Z}_{\mathrm{m}}$ contém as covariáveis pertinentes a um específico controle j, medido no tempo t da lactação. Para as medidas da persistência da lactação, as variâncias são obtidas substituindo-se os vetores $Z_{m}^{\prime}$ e $Z_{m}$ pelos vetores $Z_{i}{ }_{i}$ e $Z_{i}$ referentes ao tipo i de mensuração da persistência da lactação $\left(\mathrm{PS}_{\mathrm{i}}\right)$.

Sendo $\hat{\sigma}_{a}^{2}\left(\mathrm{PS}_{\mathrm{i}}\right)$ e $\hat{\sigma}_{p}^{2}\left(\mathrm{PS}_{\mathrm{i}}\right)$ as estimativas das variâncias genéticas aditivas e de ambiente permanente, respectivamente, associadas às medidas de persistência (PSi), as estimativas de herdabilidade, para as seis diferentes medidas da persistência da lactação $\left(\mathrm{PS}_{\mathrm{i}}\right)$, foram calculadas como exposto a seguir:

$\underline{\mathrm{PS}}_{1}$

$$
\hat{h}_{(P S 1)}^{2}=\frac{\hat{\sigma}_{a}^{2}\left(P S_{1}\right)}{\hat{\sigma}_{a}^{2}\left(P S_{1}\right)+\hat{\sigma}_{p}^{2}\left(P S_{1}\right)+\left(\hat{\sigma}_{e 1}^{2}+\hat{\sigma}_{e 6}^{2}\right)},
$$

em que o termo $\left(\hat{\sigma}_{e 1}^{2}+\hat{\sigma}_{e 6}^{2}\right)$, relativo à variância residual, foi obtido da seguinte maneira:

$\mathrm{V}_{\mathrm{e}}\left(\mathrm{PS}_{1}\right)=\mathrm{V}_{\mathrm{e}}\left(\mathrm{PL}_{240}-\mathrm{PL}_{40}\right)$, sendo que Ve indica variância residual e $\mathrm{PL}_{240}$ e $\mathrm{PL}_{40}$, respectivamente, os valores da produção de leite no dias 240 e 40;

$$
\mathrm{V}_{\mathrm{e}}\left(\mathrm{PS}_{1}\right)=\mathrm{V}_{\mathrm{e}}\left(\mathrm{PL}_{240}\right)+\mathrm{V}_{\mathrm{e}}\left(\mathrm{PL}_{40}\right)-2 \operatorname{Cov}_{\mathrm{e}}\left(\mathrm{PL}_{240}, \mathrm{PL}_{40}\right) \text {, }
$$
em que $\mathrm{V}_{\mathrm{e}}\left(\mathrm{PL}_{240}\right)$ e $\mathrm{V}_{\mathrm{e}}\left(\mathrm{PL}_{40}\right)$ são as variâncias residuais das produções de leite nos dias 240 e 40, respectivamente, e $\operatorname{Cov}_{\mathrm{e}}\left(\mathrm{PL}_{240}, \mathrm{PL}_{40}\right)$, a covariância residual entre a produção de leite nos dias 240 e 40 ; como foi assumida a independência entre os resíduos nesse trabalho, então:

$\operatorname{Cov}_{\mathrm{e}}\left(\mathrm{PL}_{240}, \mathrm{PL}_{40}\right)=0$;

portanto,

$$
\mathrm{V}_{\mathrm{e}}\left(\mathrm{PS}_{1}\right)=\mathrm{V}_{\mathrm{e}}\left(P L_{240}\right)+\mathrm{V}_{\mathrm{e}}\left(P L_{40}\right) \text {; }
$$

$\mathrm{V}_{\mathrm{e}}\left(\mathrm{PS}_{1}\right)=\hat{\sigma}_{e 6}^{2}+\hat{\sigma}_{e \mathrm{l}}^{2}$, pois o dia em lactação 240 pertence à classe de variância residual 6 (33aa a 39aㅡ semana de lactação) e o dia em lactação 40 pertence à classe de variância residual 1 (2aa $7 \underline{a}$ semana de lactação).

$\underline{\mathrm{PS}}_{2}$ $\hat{h}_{(P S 2)}^{2}=\frac{\hat{\sigma}_{a}^{2}\left(P S_{2}\right)}{\hat{\sigma}_{a}^{2}\left(P S_{2}\right)+\hat{\sigma}_{p}^{2}\left(P S_{2}\right)+\left(39 \hat{\sigma}_{e 1}^{2}+42 \hat{\sigma}_{e 2}^{2}+42 \hat{\sigma}_{e 3}^{2}+41 \hat{\sigma}_{e 4}^{2}+8 \hat{\sigma}_{e 5}^{2}\right)}$, em que o termo $\left(39 \hat{\sigma}_{e 1}^{2}+42 \hat{\sigma}_{e 2}^{2}+42 \hat{\sigma}_{e 3}^{2}+41 \hat{\sigma}_{e 4}^{2}+8 \hat{\sigma}_{e 5}^{2}\right)$, relativo à variância residual, foi obtido da seguinte forma:

$$
\begin{aligned}
& \mathrm{V}_{\mathrm{e}}\left(\mathrm{PS}_{2}\right)=\mathrm{V}_{\mathrm{e}}\left[\left(\sum_{t=96}^{181} P L_{t}\right)-\left(\sum_{t=10}^{95} P L_{t}\right)\right] ; \\
& \mathrm{V}_{\mathrm{e}}\left(\mathrm{PS}_{2}\right)=\mathrm{V}_{\mathrm{e}}\left(\sum_{t=96}^{181} P L_{t}\right)+\mathrm{V}_{\mathrm{e}}\left(\sum_{t=10}^{95} P L_{t}\right)-2 \operatorname{Cov}_{\mathrm{e}}\left(\sum_{t=96}^{181} P L_{t},\right.
\end{aligned}
$$

$\left.\sum_{t=10}^{95} P L_{t}\right)$

como os resíduos são independentes, então $\operatorname{Cov}_{\mathrm{e}}$ $\left(\sum_{t=96}^{181} P L_{t}, \sum_{t=10}^{95} P L_{t}\right)=0$, logo:

$\mathrm{V}_{\mathrm{e}}\left(\mathrm{PS}_{2}\right)=\mathrm{V}_{\mathrm{e}}\left(\sum_{t=96}^{181} P L_{t}\right)+\mathrm{V}_{\mathrm{e}}\left(\sum_{t=10}^{95} P L_{t}\right) ;$

$\mathrm{V}_{\mathrm{e}}\left(\mathrm{PS}_{2}\right)=\left[\mathrm{V}_{\mathrm{e}}\left(\mathrm{PL}_{96}+\mathrm{PL}_{97}+\ldots+\mathrm{PL}_{181}\right)\right]+\left[\mathrm{V}_{\mathrm{e}}\left(\mathrm{PL}_{10}+\mathrm{PL}_{11}\right.\right.$ $\left.\left.+\ldots+\mathrm{PL}_{95}\right)\right]$;

$\mathrm{V}_{\mathrm{e}}\left(\mathrm{PS}_{2}\right)=\left[\mathrm{V}_{\mathrm{e}}\left(\mathrm{PL}_{96}\right)+\mathrm{V}_{\mathrm{e}}\left(\mathrm{PL}_{97}\right)+\ldots+\mathrm{V}_{\mathrm{e}}\left(\mathrm{PL}_{181}\right)+7310\right.$ $\left.\operatorname{Cov}_{\mathrm{e}}\left(\mathrm{PL}_{\mathrm{t}}, \mathrm{PL}_{\mathrm{t}^{\prime}}\right)\right]+\left[\mathrm{V}_{\mathrm{e}}\left(\mathrm{PL}_{10}\right)+\mathrm{V}_{\mathrm{e}}\left(\mathrm{PL}_{11}\right)+\ldots+\mathrm{V}_{\mathrm{e}}\left(\mathrm{PL}_{95}\right)+\right.$ $\left.7310 \operatorname{Cov}_{\mathrm{e}}\left(\mathrm{PL}_{\mathrm{t}}, \mathrm{PL}_{\mathrm{t}^{\prime}}\right)\right]$; como t sempre é diferente de $\mathrm{t}^{\prime}$, então $\mathrm{Cov}_{\mathrm{e}}\left(\mathrm{PL}_{\mathrm{t}}, \mathrm{PL}_{\mathrm{t}^{\prime}}\right)=0$, logo,

$\mathrm{V}_{\mathrm{e}}\left(\mathrm{PS}_{2}\right)=\left[\mathrm{V}_{\mathrm{e}}\left(\mathrm{PL}_{96}\right)+\mathrm{V}_{\mathrm{e}}\left(\mathrm{PL}_{97}\right)+\ldots+\mathrm{V}_{\mathrm{e}}\left(\mathrm{PL}_{181}\right)\right]+\left[\mathrm{V}_{\mathrm{e}}\left(\mathrm{PL}_{10}\right)\right.$ $\left.+\mathrm{V}_{\mathrm{e}}(\mathrm{PL} 11)+\ldots+\mathrm{V}_{\mathrm{e}}\left(\mathrm{PL}_{95}\right)\right]$; mas, de acordo com as classes de variância residual usadas, $\mathrm{V}_{\mathrm{e}}\left(\mathrm{PL}_{96}\right)$ a $\mathrm{V}_{\mathrm{e}}\left(\mathrm{PL}_{132}\right)=\hat{\sigma}_{e 3}^{2}$, $\mathrm{V}_{\mathrm{e}}\left(\mathrm{PL}_{133}\right)$ a $\mathrm{V}_{\mathrm{e}}\left(\mathrm{PL}_{173}\right)=\hat{\sigma}_{e 4}^{2}, \mathrm{~V}_{\mathrm{e}}\left(\mathrm{PL}_{174)}\right.$ a $\mathrm{V}_{\mathrm{e}}\left(\mathrm{PL}_{181}\right)=\hat{\sigma}_{e 5}^{2}$, $\mathrm{V}_{\mathrm{e}}\left(\mathrm{PL}_{10}\right)$ a $\mathrm{V}_{\mathrm{e}}\left(\mathrm{PL}_{48}\right)=\hat{\sigma}_{e 1}^{2}, \mathrm{~V}_{\mathrm{e}}\left(P L_{49}\right)$ a $\mathrm{V}_{\mathrm{e}}\left(P L_{90}\right)=\hat{\sigma}_{e 2}^{2} \mathrm{e}$ $\mathrm{V}_{\mathrm{e}}\left(P L_{91}\right) \mathrm{aV}_{\mathrm{e}}\left(P L_{95}\right)=\hat{\sigma}_{e 3}^{2}, \log \mathrm{:}$

$\mathrm{V}_{\mathrm{e}}\left(\mathrm{PS}_{2}\right)=39 \hat{\sigma}_{e 1}^{2}+42 \hat{\sigma}_{e 2}^{2}+42 \hat{\sigma}_{e 3}^{2}+41 \hat{\sigma}_{e 4}^{2}+8 \hat{\sigma}_{e 5}^{2}$.

$\underline{\mathrm{PS}}_{3}$

$\hat{h}_{(P S 3)}^{2}=\frac{\hat{\sigma}_{a}^{2}\left(P S_{3}\right)}{\hat{\sigma}_{a}^{2}\left(P S_{3}\right)+\hat{\sigma}_{p}^{2}\left(P S_{3}\right)+\left(39 \hat{\sigma}_{e 1}^{2}+42 \hat{\sigma}_{e 2}^{2}+5 \hat{\sigma}_{e 3}^{2}+35 \hat{\sigma}_{e 4}^{2}+52 \hat{\sigma}_{e 5}^{2}\right)} ;$ em que o termo $39 \hat{\sigma}_{e 1}^{2}+42 \hat{\sigma}_{e 2}^{2}+5 \hat{\sigma}_{e 3}^{2}+35 \hat{\sigma}_{e 4}^{2}+52 \hat{\sigma}_{e 5}^{2}$, relativo à variância residual, foi obtido de maneira similar ao termo referente à $\mathrm{PS}_{2}$.

$\underline{\mathrm{PS}}_{4}$

$\hat{h}_{(P S 4)}^{2}=\frac{\hat{\sigma}_{a}^{2}\left(P S_{4}\right)}{\hat{\sigma}_{a}^{2}\left(P S_{4}\right)+\hat{\sigma}_{p}^{2}\left(P S_{4}\right)+\left(40008 \hat{\sigma}_{e 1}^{2}+42 \hat{\sigma}_{e 2}^{2}+42 \hat{\sigma}_{e 3}^{2}+41 \hat{\sigma}_{e 4}^{2}+43 \hat{\sigma}_{e 5}^{2}+24 \hat{\sigma}_{e 6}^{2}\right)} ;$ em que o termo:

$\left(40008 \hat{\sigma}_{e 1}^{2}+42 \hat{\sigma}_{e 2}^{2}+42 \hat{\sigma}_{e 3}^{2}+41 \hat{\sigma}_{e 4}^{2}+43 \hat{\sigma}_{e 5}^{2}+24 \hat{\sigma}_{e 6}^{2}\right)$, relativo à variância residual, foi obtido da seguinte maneira:

$\mathrm{V}_{\mathrm{e}}\left(\mathrm{PS}_{4}\right)=\mathrm{V}_{\mathrm{e}}\left[\sum_{t=41}^{240}\left(P L_{t}-P L_{40}\right)\right]$;

$\mathrm{V}_{\mathrm{e}}\left(\mathrm{PS}_{4}\right)=\mathrm{V}_{\mathrm{e}}\left[\sum_{t=41}^{240} P L_{t}-\sum_{t=41}^{240} P L_{40}\right]$ 
$\mathrm{V}_{\mathrm{e}}\left(\mathrm{PS}_{4}\right)=\mathrm{V}_{\mathrm{e}}\left(\sum_{t=41}^{240} P L_{t}\right)+\mathrm{V}_{\mathrm{e}}\left(200 \mathrm{PL}_{40}\right)-2 \operatorname{Cov}_{\mathrm{e}}$

$\left(\sum_{t=41}^{240} P L_{t}, 200 \mathrm{PL}_{40}\right)$;

$\mathrm{V}_{\mathrm{e}}\left(\mathrm{PS}_{4}\right)=\mathrm{V}_{\mathrm{e}}\left(\mathrm{PL}_{41}+\mathrm{PL}_{42}+\ldots+\mathrm{PL}_{240}\right)+(200)^{2} \mathrm{~V}_{\mathrm{e}}\left(\mathrm{PL}_{40}\right)-$ $400 \operatorname{Cov}_{\mathrm{e}}\left(\mathrm{PL}_{41}+\mathrm{PL}_{42}+\ldots+\mathrm{PL}_{240}, \mathrm{PL}_{40}\right)$;

como os resíduos são independentes, então $\operatorname{Cov}_{\mathrm{e}}\left(\mathrm{PL}_{41}{ }^{+}\right.$ $\left.\mathrm{PL}_{42}+\ldots+\mathrm{PL}_{240}, \mathrm{PL}_{40}\right)=0$, logo:

$\mathrm{V}_{\mathrm{e}}(\mathrm{PS} 4)=\mathrm{V}_{\mathrm{e}}\left(\mathrm{PL} 41+\mathrm{PL}_{42}+\ldots+\mathrm{PL}_{240}\right)+(200)^{2} \mathrm{~V}_{\mathrm{e}}\left(\mathrm{PL}_{40}\right)$;

$\mathrm{V}_{\mathrm{e}}\left(\mathrm{PS}_{4}\right)=\left[\mathrm{V}_{\mathrm{e}}\left(\mathrm{PL}_{41}\right)+\mathrm{V}_{\mathrm{e}}\left(\mathrm{PL}_{42}\right)+\ldots+\mathrm{V}_{\mathrm{e}}\left(\mathrm{PL}_{240}\right)+39800\right.$

$\left.\operatorname{Cov}_{\mathrm{e}}\left(\mathrm{PL}_{\mathrm{t}}, \mathrm{PL}_{\mathrm{t}^{\prime}}\right)\right]+40000 \mathrm{~V}_{\mathrm{e}}\left(\mathrm{PL}_{40}\right)$; mas como t sempre é diferente de t', então $\operatorname{Cov}_{\mathrm{e}}\left(\mathrm{PL}_{\mathrm{t}}, \mathrm{PL}_{\mathrm{t}^{\prime}}\right)=0$, logo:

$\mathrm{V}_{\mathrm{e}}\left(\mathrm{PS}_{4}\right)=\mathrm{Ve}\left(\mathrm{PL}_{41}\right)+\mathrm{Ve}\left(\mathrm{PL}_{42}\right)+\ldots+\mathrm{Ve}\left(\mathrm{PL}_{240}\right)+$ $40000 \mathrm{~V}_{\mathrm{e}}\left(\mathrm{PL}_{40}\right)$; mas, de acordo com as classes de variância residual usadas, $\mathrm{V}_{\mathrm{e}}\left(\mathrm{PL}_{41}\right)$ a $\mathrm{V}_{\mathrm{e}}\left(\mathrm{PL}_{48}\right)=\hat{\sigma}_{e l}^{2}, \mathrm{~V}_{\mathrm{e}}\left(\mathrm{PL}_{49}\right)$ a $\mathrm{V}_{\mathrm{e}}\left(\mathrm{PL}_{90}\right)=\hat{\sigma}_{e 4}^{2}, \mathrm{~V}_{\mathrm{e}}\left(\mathrm{PL}_{91}\right)$ a $\mathrm{V}_{\mathrm{e}}\left(\mathrm{PL}_{132}\right)=\hat{\sigma}_{e 2}^{2}, \mathrm{~V}_{\mathrm{e}}\left(\mathrm{PL}_{133}\right) \mathrm{a}$ $\mathrm{V}_{\mathrm{e}}\left(\mathrm{PL}_{173}\right)=\hat{\sigma}_{e 4}^{2}, \mathrm{~V}_{\mathrm{e}}\left(\mathrm{PL}_{174}\right)$ a $\mathrm{V}_{\mathrm{e}}\left(\mathrm{PL}_{216}\right)=\hat{\sigma}_{e 5}^{2} \mathrm{e} \mathrm{V}_{\mathrm{e}}\left(\mathrm{PL}_{217}\right)$ a $\mathrm{V}_{\mathrm{e}}\left(\mathrm{PL}_{240}\right)=\hat{\sigma}_{e 6}^{2}$, logo:

$\mathrm{V}_{\mathrm{e}}\left(\mathrm{PS}_{4}\right)=40008 \hat{\sigma}_{e 1}^{2}+42 \hat{\sigma}_{e 2}^{2}+42 \hat{\sigma}_{e 3}^{2}+41 \hat{\sigma}_{e 4}^{2}+43 \hat{\sigma}_{e 5}^{2}+24 \hat{\sigma}_{e 6}^{2}$. $\underline{\mathrm{PS}}_{5}$

$\hat{h}_{(P S S)}^{2}=\frac{\hat{\sigma}_{a}^{2}\left(P S_{5}\right)}{\hat{\sigma}_{a}^{2}\left(P S_{5}\right)+\hat{\sigma}_{p}^{2}\left(P S_{5}\right)+\left(9 \hat{\sigma}_{e 1}^{2}+42 \hat{\sigma}_{e 2}^{2}+42 \hat{\sigma}_{e 3}^{2}+41 \hat{\sigma}_{e 4}^{2}+43 \hat{\sigma}_{e 5}^{2}+40023 \hat{\sigma}_{e 6}^{2}\right)}$; em que o termo $\left(9 \hat{\sigma}_{e 1}^{2}+42 \hat{\sigma}_{e 2}^{2}+42 \hat{\sigma}_{e 3}^{2}+41 \hat{\sigma}_{e 4}^{2}+43 \hat{\sigma}_{e 5}^{2}+40023 \hat{\sigma}_{e 6}^{2}\right)$, relativo à variância residual, foi obtido de maneira similar ao termo referente à $\mathrm{PS}_{4}$.

$\underline{\mathrm{PS}}_{6}$

$$
\hat{h}_{(P S 6)}^{2}=\frac{\hat{\sigma}_{a}^{2}\left(P S_{6}\right)}{\hat{\sigma}_{a}^{2}\left(P S_{6}\right)+\hat{\sigma}_{p}^{2}\left(P S_{6}\right)+\left(\hat{\sigma}_{e 2}^{2}+\hat{\sigma}_{e 6}^{2}\right)} ;
$$

em que o termo $\left(\hat{\sigma}_{e 2}^{2}+\hat{\sigma}_{e 6}^{2}\right)$, relativo à variância residual, foi obtido de maneira similar ao termo referente à PS ${ }_{1}$.

A estimativa da herdabilidade para produção de leite até 268 dias de lactação (P268) foi calculada por:

$\hat{h}_{(P 268)}^{2}=\frac{\hat{\sigma}_{a}^{2}(P 268)}{\hat{\sigma}_{a}^{2}(P 268)+\hat{\sigma}_{p}^{2}(P 268)+\left(39 \hat{\sigma}_{e 1}^{2}+42 \hat{\sigma}_{e 2}^{2}+42 \hat{\sigma}_{e 3}^{2}+41 \hat{\sigma}_{e 4}^{2}+43 \hat{\sigma}_{e S}^{2}+52 \hat{\sigma}_{e 6}^{2}\right)}$

As estimativas de correlações genéticas foram calculadas, respectivamente, por:

$$
r g_{k n}=\frac{\hat{\sigma}_{a_{k n}}}{\sqrt{\hat{\sigma}_{a_{k k}}^{2} * \hat{\sigma}_{a_{n n}}^{2}}}
$$

em que $\mathrm{k}$ e $\mathrm{n}$ podem representar as seis medidas de persistência da lactação $\left(\mathrm{PS}_{\mathrm{i}}\right)$, a produção de leite na lactação (P268) e as produções no dia do controle aos 10 (C10), 35 (C35), 135 (C135) e 240 (C240) dias de lactação.

\section{Resultados e Discussão}

O modelo de regressão aleatória gerou estimativas das matrizes de (co) variâncias dos coeficientes de regressão aleatória, atribuídas aos efeitos genéticos e de ambiente permanente (Tabela 2), e estimativas das variâncias residuais para as seis classes criadas ao longo da lactação iguais, respectivamente, a 0,1730; 0,1706; 0,1416; 0,1244; 0,0946 e $0,1177 \mathrm{~kg}^{2}$.

As estimativas de herdabilidade, para as diferentes medidas de persistência, variaram de 0,03 a 0,09 (Tabela 3). A utilização de animais provenientes de um único rebanho e, consequentemente, de base genética semelhante pode explicar os baixos valores de herdabilidade encontrados. Apesar de os parâmetros genéticos serem específicos da espécie, raça ou mesmo da população com que se está trabalhando, buscaram-se trabalhos com persistência da lactação de caprinos como base para comparações com os resultados obtidos neste estudo. Todavia, nenhum foi encontrado na literatura consultada. Para bovinos de leite, encontrou-se larga amplitude de estimativas de herdabilidade $(0,00$ a 0,40$)$ para diferentes medidas de persistência da lactação obtidas por meio de diferentes modelos de regressão aleatoria (Gengler et al., 1999; Jamrozik et al., 2000; Cobuci et al., 2004; Cobuci et al., 2006a; Cobuci et al., 2006b; Cobuci et al., 2007).

Segundo Cobuci et al. (2004), diferenças entre herdabilidades para persistência da lactação podem estar associadas à parte da lactação utilizada no cálculo da persistência. Portanto, medidas de persistência que utilizam períodos da lactação, cujas produções no dia do controle tenham maiores herdabilidades, tendem, também, a ter maiores herdabilidades, o que pode conduzir à falsa impressão de que a melhor medida para se avaliar persistência da lactação seja aquela calculada usando períodos de lactação com altas herdabilidades. Contudo, a escolha de um critério de seleção deve ser baseada, primeiramente, na adequação ao objetivo de seleção, e não em sua herdabilidade, apesar da importância desta para o sucesso do processo seletivo.

As estimativas de correlações genéticas entre as medidas de persistência analisadas variaram de -1,00 a 1,00 (Tabela 3) e evidenciam grande diversidade de graus de associação entre as medidas. Esses resultados estão de

Tabela 2 - Estimativas de covariâncias genéticas $\left(\hat{K}_{a}\right)$ e de ambiente permanente ( $\hat{K}_{p}$ ) para os coeficientes de regressão aleatória, obtidas por modelo de regressão aleatória

$$
\begin{gathered}
\hat{K}_{a}=\left[\begin{array}{lll}
\hat{g}_{11} & \hat{g}_{12} & \hat{g}_{13} \\
\hat{g}_{21} & \hat{g}_{22} & \hat{g}_{23} \\
\hat{g}_{31} & \hat{g}_{32} & \hat{g}_{33}
\end{array}\right]=\left[\begin{array}{rll}
0,28 & -0,63 \times 10^{-3} & -0,29 \\
-0,63 \times 10^{-3} & 0,19 \times 10^{-5} & 0,68 \times 10^{-3} \\
-0,29 & 0,68 \times 10^{-3} & 0,37
\end{array}\right] \\
\hat{K}_{p}=\left[\begin{array}{llll}
\hat{p}_{11} & \hat{p}_{12} & \hat{p}_{13} \\
\hat{p}_{21} & \hat{p}_{22} & \hat{p}_{23} \\
\hat{p}_{31} & \hat{p}_{32} & \hat{p}_{33}
\end{array}\right]=\left[\begin{array}{rll}
0,73 & -0,25 \times 10^{-2} & -0,72 \\
-0,25 \times 10^{-2} & 0,13 \times 10^{-4} & 0,28 \times 10^{-2} \\
-0,72 & 0,28 \times 10^{-2} & 1,38
\end{array}\right]
\end{gathered}
$$

Os índices 1, 2 e 3 referem-se aos coeficientes de regressão aleatória $a_{1}$, a $a_{2}$ e $a_{3}$ da função de Wilmink modificada. 
Tabela 3 - Estimativas de herdabilidade (diagonal principal) e correlações genéticas para as medidas de persistência da lactação

\begin{tabular}{lcccccc}
\hline Característica* & $\mathrm{PS}_{1}$ & $\mathrm{PS}_{2}$ & $\mathrm{PS}_{3}$ & $\mathrm{PS}_{4}$ & $\mathrm{PS}_{5}$ & $\mathrm{PS}_{6}$ \\
\hline $\mathrm{PS}_{1}$ & 0,05 & 0,62 & 1,00 & 0,80 & $-0,91$ & 0,93 \\
$\mathrm{PS}_{2}$ & & 0,09 & 0,67 & 0,96 & $-0,24$ & 0,29 \\
$\mathrm{PS}_{3}$ & & & 0,08 & 0,84 & $-0,88$ & 0,90 \\
$\mathrm{PS}_{4}$ & & & & 0,03 & $-0,49$ & 0,53 \\
$\mathrm{PS}_{5}$ & & & & 0,06 & $-1,00$ \\
$\mathrm{PS}_{6}$ & & & & & 0,06 \\
${ }^{*} \mathrm{PS}_{1}$ a $\mathrm{PS}_{6}$ - medidas da persistência da lactação. & & &
\end{tabular}

acordo com os encontrados por Cobuci et al. (2004) em bovinos da raça Holandesa. Esses autores encontraram estimativas de correlações genéticas entre as seis medidas de persistência avaliadas nesse estudo, porém sem adaptações para caprinos (medidas originais), que variaram de $-0,99$ a 0,99 . As maiores estimativas, 1,00 e $-1,00$, foram encontradas entre as medidas $\mathrm{PS}_{1}$ e $\mathrm{PS}_{3}$, e $\mathrm{PS}_{5}$ e $\mathrm{PS}_{6}$, respectivamente, enquanto as menores foram encontradas entre a medida $\mathrm{PS}_{2}$ e as medidas $\mathrm{PS}_{5}$ e $\mathrm{PS}_{6}$, de -0,24 e 0,29, respectivamente. Estimativas de correlação genética negativas entre a $\mathrm{PS}_{5}$ e as demais medidas foram observadas, possivelmente pelo fato de essas medidas serem antagônicas, ou seja, maiores valores de $\mathrm{PS}_{5}$ indicam maior persistência da lactação, enquanto o mesmo é observado para menores valores das demais medidas.

As estimativas das correlações genéticas entre as medidas de persistência e produção de leite até 268 dias variaram de -0,64 a 0,67 (Tabela 4). Em bovinos de leite, as estimativas de correlações genéticas entre as medidas de persistência e produção de leite até 305 dias variaram de -0,31 a 0,55 (Jakobsen et al., 2002; Cobuci et al., 2004). As medidas $\mathrm{PS}_{4}$ e $\mathrm{PS}_{5}$ apresentaram, respectivamente, a menor e a maior correlação genética com produção de leite até 268 dias $(0,14$ e 0,67$)$. O baixo valor de correlação genética entre $\mathrm{PS}_{4}$ e $\mathrm{P}_{268}(0,14)$ indica a possibilidade de se alterar o formato da curva de lactação de uma cabra sem provocar alterações na produção de leite.

Correlações genéticas negativas das produções de leite aos 10 e 30 dias de lactação com as medidas $\mathrm{PS}_{1}, \mathrm{PS}_{2}$, $\mathrm{PS}_{3}, \mathrm{PS}_{4}$ e $\mathrm{PS}_{6}$ e positivas com a medida $\mathrm{PS}_{5}$ (Tabela 4) são indicativos de que as cabras com maior produção no início da lactação também são as mais persistentes. Isso, a princípio, contradiz Lean et al. (1989), que relataram que animais com maiores produções no início da lactação tendem a ter um declínio mais acentuado da produção de leite (menor persistência), quando comparados aos de menores produções. Essa discordância entre os resultados pode ser explicada pelo fato de o rebanho caprino, usado no presente estudo, não apresentar nível de produção muito alto (Tabela 1), não sofrendo de maneira severa, portanto, problemas de balanço energético negativo e, consequentemente, de doenças metabólicas, que poderiam ser causa da relação antagônica entre produção de leite no pico e persistência da lactação. Segundo Capuco et al. (2003), práticas de manejo que garantam a manutenção do número de células mamárias ao longo da lactação aumentam a persistência, o que pode explicar os resultados encontrados, uma vez que cabras de maior produção normalmente recebem tratamento superior. Outra hipótese é que cabras com maiores produções no início da lactação tenham tido reconcepção mais tardia comparadas àquelas de menor produção, sofrendo, portanto, menor influência da gestação, processo este intimamente ligado à redução da produção de leite ao longo da lactação.

As correlações genéticas entre as produções de leite aos 10, 30, 135 e 240 dias de lactação com produção acumulada até os 268 dias da lactação foram altas (Tabela 4) e confirmam pesquisas de Jakobsen et al. (2002) com bovinos da raça Holandesa. Esses altos valores podem ser explicados pelo fato de o valor genético da produção acumulada até os 268 dias da lactação ser obtido por somatório que inclui os valores genéticos para as produções aos 10, 30, 135 e 240 dias da lactação. Essas correlações altas também sugerem grande coincidência em cabras selecionadas usando como critério de seleção valores genéticos para as produções no dia do controle aos 30, 135 e 240 dias da lactação e para produção acumulada até os 268 dias da lactação.

As estimativas de herdabilidade foram menores para produções no início e ao final da lactação (aos 10 e 240 dias) e maiores no meio desta (aos 135 dias da lactação). Segundo Assis et al. (2006), esses resultados podem indicar menor

Tabela 4 - Estimativas de herdabilidade e de correlações genéticas entre medidas de persistência da lactação e a produção de leite nos períodos selecionados da lactação e a produção de leite até 268 dias

\begin{tabular}{lccrrrrrr}
\hline Produção de leite & Herdabilidade & PS $_{1}$ & PS $_{2}$ & PS $_{3}$ & PS $_{4}$ & PS $_{5}$ & $-0,51$ & 0,52 \\
\hline Aos 10 dias de lactação & 0,07 & $-0,59$ & $-0,41$ & $-0,58$ & $-0,53$ & 0,61 \\
Aos 30 dias de lactação & 0,16 & $-0,62$ & 0,06 & $-0,57$ & $-0,16$ & 0,79 & $-0,78$ & 0,95 \\
Aos 135 dias de lactação & 0,25 & $-0,41$ & 0,37 & $-0,34$ & 0,15 & 0,69 & $-0,67$ & 1,00 \\
Aos 240 de lactação & 0,17 & $-0,10$ & 0,56 & $-0,03$ & 0,39 & 0,41 & $-0,38$ & 0,95 \\
Até os 268 dias de lactação & 0,31 & $-0,39$ & 0,36 & $-0,33$ & 0,14 & 0,67 \\
\hline
\end{tabular}

$\mathrm{h}^{2}$ = herdabilidade; $\mathrm{PS}_{1}$ a $\mathrm{PS}_{6}=$ persistência da lactação; $\mathrm{P}_{268}=$ produção de leite acumulada até 268 dias de lactação. 
influência genética na variância total ao início e final da lactação. A estimativa de herdabilidade para produção acumulada até os 268 dias, de 0,31 (Tabela 4), foi inferior à encontrada por Sarmento et al. (2006), de 0,43 para produção de leite até 270 dias em cabras da raça Alpina, e superior à registrada por Delgado et al. (2006), de 0,14 para produção de leite até 240 dias em cabras da raça Murciano-Granadina, utilizando modelo animal multicaracterístico, com dados repetidos. Não foram encontrados na literatura estudos com estimativa de herdabilidade para produção de leite até 240 ou 270 dias utilizando modelo de regressão aleatoria em caprinos. Contudo, o valor de herdabilidade encontrado neste estudo sugere boa resposta à seleção para produção de leite no rebanho.

Segundo Jakobsen et al. (2002), a medida ideal para persistência da lactação deve apresentar valor econômico expressivo, elevada variância genética, alta herdabilidade e baixa correlação genética com a produção total de leite até 305 dias (268 dias neste estudo). A medida de persistência da lactação deve ter baixa correlação genética com produção acumulada até os 268 dias da lactação, caso contrário, não seria necessário praticar seleção para persistência; seria suficiente selecionar para produção de leite, como normalmente é feito. Logo, como o objetivo de seleção, neste caso, é alterar o formato da curva de lactação sem alterar a produção de leite total, o principal critério adotado para a escolha da medida de persistência a ser usada foi apresentar mínima correlação genética com produção de leite acumulada até os 268 dias da lactação. Portanto, sugere-se que a medida $\mathrm{PS}_{4}$ seja escolhida para uso em avaliações genéticas da persistência da lactação de cabras Saanen no rebanho estudado. No entanto, devido à baixa herdabilidade estimada para essa característica, deve-se esperar que a seleção para persistência da lactação apresente baixa resposta.

\section{Conclusões}

As medidas de persistência da lactação avaliadas foram variáveis quanto à adequação ao objetivo de seleção, que é modificar o formato da curva de lactação sem causar mudanças na produção de leite total. Algumas apresentaram alta correlação genética com produção de leite até 268 dias, logo seu uso seria desnecessário, pois a seleção para produção já seria suficiente. No entanto, obtiveram-se medidas que se adéquam ao objetivo e, portanto, podem ser utilizadas em avaliações genéticas de cabras Saanen para persistência da lactação. Entretanto, para o rebanho estudado, as respostas à seleção para persistência da lactação devem ser pequenas, em decorrência da pequena herdabilidade estimada para essa característica.

\section{Referências}

ASSIS, G.M.L.; ALBUQUERQUE, L.G.; SARMENTO, J.L.R. et al. Estimação de parâmetros genéticos em caprinos leiteiros por meio de análise de regressão aleatória utilizando-se a Amostragem de Gibbs. Revista Brasileira de Zootecnia, v.35, n.3, p.706-714, 2006.

CAPUCO, A.V.; ELLIS, S.E.; HALE, S.A. et al. Lactation persistency: Insights from mammary cell proliferation studies. Journal of Animal Science, v.81, n.3, p.18-31, 2003.

COBUCI, J.A.; EUCLYDES, R.F.; PEREIRA, C.S. et al. Persistência da lactação - uma revisão. Archivos Latinoamericanos de Producción Animal, v.11, n.3, p.163-173, 2003.

COBUCI, J.A.; EUCLYDES, R. F.; COSTA, C.N. et al. Análises da persistência na lactação de vacas da raça holandesa, usando produção no dia do controle e modelo de regressão aleatória. Revista Brasileira de Zootecnia, v.33, n.2, p.546-554, 2004.

COBUCI, J.A.; COSTA, C.N.; FREITAS, A.F. et al. Genetic parameters of milk yield persistency of Holstein cows in Brazil. In: WORLD CONGRESS GENETIC APPLIED LIVESTOCK PRODUCTION, 8., 2006, Belo Horizonte. Proceedings.. Belo Horizonte, 2006a. (CD-ROM).

COBUCI, J.A.; COSTA, C.N.; TEIXEIRA, N.M. et al. Utilização dos polinômios de Legendre e da função de Wilmink em avaliações genéticas para persistência na lactação de animais da raça Holandesa. Arquivo Brasileiro de Medicina Veterinária e Zootecnia, v.58, n.4, p.614-623, 2006b.

COBUCI, J.A.; EUCLYDES, R.F.; COSTA, C.N. et al. Genetic evaluation for persistency of lactation in Holstein cows using a random regression model. Genetics and Molecular Biology, v.30, n.2, p.349-355, 2007.

DELGADO, J.V.; LEÓN, J.M.; GAMA, L.T. et al. Genetic parameters for milk traits in Murciano-Granadina goats in the high lands. In: WORLD CONGRESS GENETIC APPLIED LIVESTOCK PRODUCTION, 8., 2006, Belo Horizonte. Proceedings... Belo Horizonte, 2006. (CD-ROM).

GENGLER, N.; TIJANI, A.; WIGGANS, G.R. et al. Estimation of (co)variance functions of test day yields in first and later lactation of United States cows. Interbull Bulletin, n.22, p.69-73, 1999.

HARDER B.; BENNEWITZ J.; HINRICHS D. et al. Genetic parameters for health traits and their relationship to different persistency traits in German Holstein dairy cattle. Journal of Dairy Science, v.89, n.8, p.3202-3212, 2006.

HERRERA, L.G.G.; EL FARO, L.; ALBUQUERQUE, L.G. et al. Estimativas de parâmetros genéticos para produção de leite e persistência da lactação em vacas Gir, aplicando modelos de regressão aleatória. Revista Brasileira de Zootecnia, v.37, n.9, p.1584-1594, 2008

JAKOBSEN, J.H.; MADSEN, P.; JENSEN, J. et al. Genetic parameters for milk production and persistency for Danish Holstein estimated in random regression models using REML. Journal of Dairy Science, v.85, n.6, p.1607-1616, 2002.

JAMROZIK, J.; SCHAEFFER. L.R.; DEKKERS, J.C.M. Genetic evaluation of dairy cattle using test day yields and random regression model. Journal of Dairy Science, v.80, n.6, p.1217-1226, 1997.

JAMROZIK, J.; JANSEN, G.; SCHAEFFER, L.R. et al. Analysis of persistency of lactation calculates from a random regression test day model. Interbull Bulletin, n.17, p.64-69, 1998.

JAMROZIK, J.; SCHAEFFER, R.L.; JANSEN, G.B. Approximate accuracies of prediction from random regression models. Livestock Production Science, v.66, p.85-92, 2000. 
LEAN, I.J.; GALLAND, J.C; SCOTT, J.L. Relationships between fertility, peak milk yields and lactational persistency in dairy cows. Theriogenology, v.31, n.5, p.1093-1103, 1989.

MENEZES, G.R.O. Avaliação da produção de leite em cabras da raça Saanen utilizando modelos de regressão aleatória. 2008. 79f. Dissertação (Mestrado) - Universidade Federal de Viçosa, Viçosa, MG.

MEYER, K. DXMRR - A program to estimate covariance functions for longitudinal data by REML. In: WORLD CONGRESS OF GENETICS APPLIED TO LIVESTOCK PRODUCTION, 6.,
1998, Armidale. Proceedings... Armidale: University of New England, 1998. (CD-ROM).

SARMENTO, J.L.R.; REIS FILHO, J.C.; ALBUQUERQUE, L.G. et al. Avaliação genética de caprinos da raça Alpina utilizandose a produção de leite no dia do controle. Revista Brasileira de Zootecnia, v.35, n.2, p.443-451, 2006.

SÖLKNER, J.; FUCHS, W. A comparison of different measures of persistency with special respect to variation of test-day milk yields. Livestock Production Science, v.16, p.305-319, 1987. 